\title{
Anaphylactic shock: no time to think
}

\author{
${ }^{1} \mathrm{M}$ Whiteside, ${ }^{2} \mathrm{~A}$ Fletcher \\ ${ }^{1}$ Specialist Registrar in Acute Medicine; ${ }^{2}$ Consultant Acute and Emergency Physician, Sheffield Teaching Hospitals, Sheffield, UK
}

\begin{abstract}
Anaphylaxis is the quintessential medical emergency where prompt recognition and treatment is life-saving. In the UK the incidence is increasing year on year, and is most common in the sixth and seventh decades of life. More than half of cases are iatrogenic in nature, most of the rest are caused by venom (stings) and food substances. The clinical signs can be subtle, but an acute onset of skin or mucosal oedema with respiratory compromise or reduced blood pressure should alert the physician to the diagnosis. The management revolves around the use of adrenaline after an initial airway, breathing and circulation approach, in a dose of $0.5 \mathrm{mg}$ I:I,000 intramuscularly, repeated five minutes later if there has been no response. Any delay in treatment is associated with increased risk of adverse outcome. Steroids and antihistamines are often given, although there is no convincing evidence of their effect in the acute setting. Where diagnostic uncertainty arises, serum tryptase levels can confirm or refute the diagnosis.
\end{abstract}

KEYWORDS Adrenaline, anaphylactoid, anaphylaxis, shock

DECLARATION OF INTERESTS No conflict of interests declared.
Correspondence to $M$ Whiteside, Department of Acute \& Emergency Medicine, Northern General Hospital, Herries Road, Sheffield S5 7AU, UK

tel. +44 (0)II 142715769 e-mail mike.whiteside@sth.nhs.uk

\section{INTRODUCTION}

Anaphylaxis is the quintessential medical emergency where prompt intervention can be life-saving. It is defined as 'a serious allergic reaction that is rapid in onset and may cause death'.' Much has been made of the differences between anaphylaxis (immunoglobulin $\mathrm{E}[\mathrm{lgE}]$ mediated) and anaphylactoid reactions, the consensus now being that the two are identical from a treatment point of view, so the term anaphylactoid is redundant. For this article anaphylaxis will mean both lgE- and nonIgE-mediated reactions. The severity of anaphylaxis can vary greatly from a mild urticarial rash coming on over a period of hours to airway obstruction by oedema within minutes. It is these acute reactions where prompt recognition and consideration of the diagnosis saves lives. However, it has been shown that among medical staff there remains confusion over the correct dose and route of administration of adrenaline. ${ }^{2}$

\section{BACKGROUND}

- The true rate of anaphylaxis is unknown due to under-reporting, occurrences outside health facilities, lack of consideration of the diagnosis and the definition used.

- Data for England in 2005 show a lifetime prevalence of 75 per 100,000 patients, increasing year on year. ${ }^{3}$

- Up to the age of 15, males are more likely to be affected, but this likelihood is reversed after that.

- Anaphylaxis is most common in the sixth and seventh decades of life.

- Trigger factors change with age (food allergy is more likely in adolescents and young adults). The rate also increases in good socio-economic circumstances,

TABLE I Signs associated with anaphylaxis
\begin{tabular}{|l|l|}
\hline Cardiovascular system & Tachycardia, hypotension \\
\hline Respiratory system & $\begin{array}{l}\text { Bronchospasm, laryngeal } \\
\text { oedema, stridor }\end{array}$ \\
\hline Skin & Urticaria, erythema, angio-oedema \\
\hline Facial & $\begin{array}{l}\text { Facial oedema, rhinitis, } \\
\text { conjunctival oedema }\end{array}$ \\
\hline Others & $\begin{array}{l}\text { Diarrhoea and vomiting, } \\
\text { metallic taste, confusion }\end{array}$ \\
\hline
\end{tabular}

TABLE 2 Causes of anaphylaxis

\begin{tabular}{|l|l|}
\hline Drugs & $\begin{array}{l}\text { Non-steroidal anti-inflammatories } \\
\text { (NSAIDS), aspirin, antibiotics, colloids, } \\
\text { vitamin K, contrast media }\end{array}$ \\
\hline Blood products & Packed cells, immunoglobulins, vaccines \\
\hline Foods & Eggs, peanuts, shellfish \\
\hline Stings & Bee and wasp stings \\
\hline
\end{tabular}

presumably because this exposes the population to drugs used in healthcare. ${ }^{4}$

Anaphylaxis occurs due to the degranulation of basophils and mast cells releasing such mediators as histamine, tryptase and interleukins. It may occur due to re-exposure of pre-sensitised cells to the allergen (via $\lg \mathrm{E}$ ) or via another non-immunological mechanism, but clinically the two mechanisms are indistinct. These chemicals lead directly to the signs associated with anaphylaxis (see Table I).

The most common signs and symptoms are urticaria (73\%), itch (43\%) and dyspnoea (29\%), with hypotension only occurring in around $10 \%$ of cases. ${ }^{5}$ 
TABLE 3 Clinical criteria for anaphylaxis

Anaphylaxis is highly likely when any one of the following three criteria is fulfilled:

Acute onset of an illness (minutes to several hours) with involvement of the skin, mucosal tissue or both (e.g. generalised hives, pruritus or flushing, swollen lips/ tongue/uvula) and at least one of the following:

- Respiratory compromise (e.g. dyspnoea, wheeze/bronchospasm, stridor, reduced peak expiratory flow [PEF], hypoxaemia)

- Reduced blood pressure (BP $<90 \mathrm{mmHg}$ systolic) or associated symptoms of end-organ dysfunction (e.g. hypotonia [collapse], syncope)

Two or more of the following that occur rapidly after exposure to a likely allergen for that patient (minutes to several hours)

- Involvement of the skin/mucosal tissue (e.g. generalised hives, itch/flush, swollen lips/tongue/uvula)

- Respiratory compromise (e.g. dyspnoea, wheeze/bronchospasm, stridor, reduced PEF, hypoxaemia)

- Reduced blood pressure or associated symptoms

(e.g. hypotonia, syncope)

- Persistent gastrointestinal symptoms (e.g. crampy abdominal pain, vomiting)

Reduced blood pressure after exposure to known allergen for that patient (minutes to several hours)

- Infants and children: low systolic BP (age specific) or greater than $30 \%$ decrease in systolic BP

- Adults: systolic BP of less than $90 \mathrm{mmHg}$ or greater than $30 \%$ decrease from that person's baseline (e.g. patients with hypertension dropping their BP may still be $>90 \mathrm{mmHg}$ ).

\section{CAUSES OF ANAPHYLACTIC SHOCK}

In the UK, half of all anaphylactic shock has iatrogenic causes. ${ }^{6}$ Almost any substance can cause anaphylaxis, but stings (venom) and food account for the vast majority of non-drug causes (see Table 2).

\section{DIAGNOSIS}

The key to diagnosing anaphylaxis is recognising the signs, thus allowing it to be considered. The classic patient presents with rapidly progressive signs with skin and respiratory involvement (versus sepsis, panic attack and asthma as differential diagnoses). There may often be a history of recent allergen exposure or previous episodes. However, patients can also present more insidiously, so careful thought needs to be given to symptoms of incontinence, collapse and confusion. Skin signs should be actively looked for because mild erythema may be all that is present initially. An algorithm may help (see Table 3).

It should be remembered that hypotension and rash may also be a sign of sepsis (especially meningococcal sepsis).

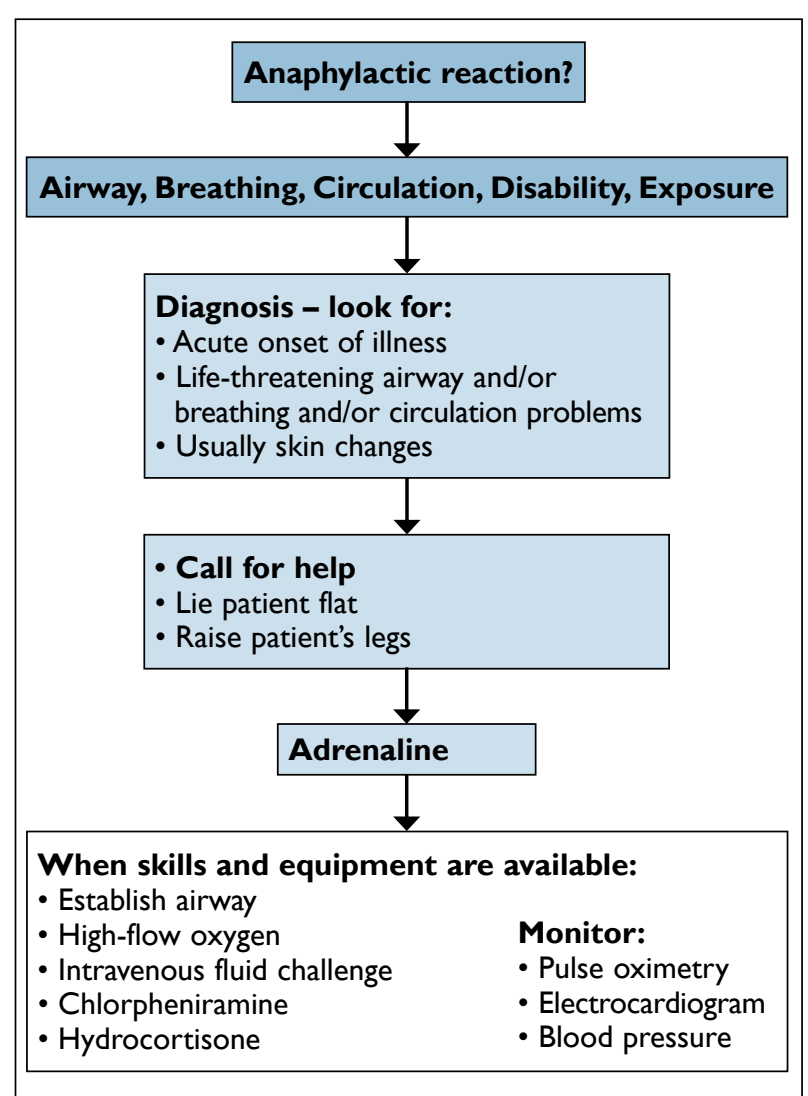

FIGURE I Anaphylaxis algorithm, from Soar J et al.

'Emergency treatment of anaphylactic reactions - guidelines for healthcare providers"

\section{MANAGEMENT}

As with any emergency, a rapid airway, breathing and circulation $(A B C)$ assessment is necessary, but ' $A$ ' should also include 'allergen removal'. If any of the three criteria above are met, then immediate treatment with intramuscular (IM) adrenaline should be given, using 0.5 $\mathrm{mg}$, I in 1,000 , injected into the mid-anterolateral thigh. Confusion exists due to the different dose and mode of delivery versus that used in arrest situations, with only $16.8 \%$ of junior doctors getting it right. ${ }^{2}$ The UK resuscitation guidelines ${ }^{7}$ (see Figure I) give a very clear management algorithm. There will be occasions, when a patient may present and not meet the criteria above, where prompt use of adrenaline may be justified, such as proven anaphylaxis to egg products where egg has just been eaten and the patient has urticaria. Delay in the administration of adrenaline is associated with more adverse outcomes (death); the time from exposure to first cardiac arrest can be as little as one minute with intravenous drugs. ${ }^{7,8}$

Oxygen should be administered ${ }^{9}$ and if the patient shows signs of shock they should be laid flat with the legs elevated to help increase cardiac output. Obtain intravenous access and give a fluid challenge (e.g. 500$\mathrm{I}, 000 \mathrm{ml}$ crystalloid). In the first few minutes up to $35 \%$ of the circulating volume may extravasate, plus there will 
be vasodilatation, so large volumes of crystalloid or colloid are often required. ${ }^{10}$ There is no evidence as to the best fluid to give, although if anaphylaxis occurred while the patient was receiving a colloid infusion, that should be considered a potential cause and stopped, to be replaced with crystalloid.

The response should be tailored to the situation: a predominantly respiratory reaction will not require such aggressive fluid resuscitation. If after two doses of intramuscular adrenaline the patient is not improving, critical care input (i.e. an anaesthetic review) should be sought. It is only in this setting that intravenous adrenaline can be used by experienced specialists (side effects are arrhythmias, myocardial infarction and stroke). Severe ischaemic heart disease and stroke are relative contraindications to the use of adrenaline, but in a situation with shock their benefit will outweigh the risks. If the patient has a cardiorespiratory arrest, then advanced life support procedures should be followed.

Bronchospasm may respond to nebulised beta-agonists, and if the reaction has features of asthma alone then the British Thoracic Society (BTS) asthma guidelines" should be followed. It should be noted that intravenous magnesium is a vasodilator and can cause hot flushes and make hypotension worse. ${ }^{7}$

The use of corticosteroids in other forms of allergy is known to be beneficial so they are included in the treatment algorithm. The onset of action is too slow to have an effect in the acute setting. Antihistamines, while in the algorithm, have no clear evidence of benefit or harm and the onset of action will be delayed by at least an hour. ${ }^{12}$ They will only help to relieve urticaria and pruritis, thus having no effect on hypovolaemia or respiratory symptoms.

Investigations should be ordered as with any medical emergency, including an electrocardiogram, a full set of bloods and blood gases if indicated (see BTS emergency oxygen guidelines ${ }^{9}$ ). Where there is diagnostic uncertainty tests can be done looking for the mediators of the reaction. Histamine has a short half-life and is difficult to process, so the serum tryptase level (contained in mast cell granules) taken in the first three hours will usually be elevated, thus confirming the clinical diagnosis. After six hours and before 30 minutes post-event, serum tryptase may be undetectable. If possible three samples should be sent, at 30 minutes, two hours and after six hours so a baseline level and documented rise in titre can be seen. ${ }^{13}$

Once the patient has stabilised, a period of observation (at least six hours) is required since up to $20 \%$ of patients will have a biphasic response and will relapse. In our experience, such effects are difficult to differentiate from the 'wearing off' of the initial dose(s) of adrenaline and seem more common when the allergen has been ingested (with continued exposure). ${ }^{1,13}$ Before discharge, patients should be reviewed by a senior physician and all patients with a severe reaction should be referred to the local allergy clinic with detailed notes describing the clinical episode. From here skin-prick testing, advice and the provision of emergency adrenaline injections can be given.

\section{REFERENCES}

I Sampson HA, Muñoz-Furlong A, Campbell RL et al. Second symposium on the definition and management of anaphylaxis: summary report - Second National Institute of Allergy and Infectious Disease/Food Allergy and Anaphylaxis Network symposium. J Allergy Clin Immunol 2006; I 17:39I-7. doi:I0.1016/j. jaci.2005.12.1303

2 Jose R, Clesham GJ. Survey of the use of epinephrine (adrenaline) for anaphylaxis by junior hospital doctors. Postgrad Med J 2007; 83:6I0-II. doi:I0.1I36/pgmj.2007.059097

3 Sheikh A, Hippisley-Cox J, Newton J et al. Trends in national incidence, lifetime prevalence and adrenaline prescribing for anaphylaxis in England.J R Soc Med 2008; I0I:I39-43. doi:I0.1258/ irsm.2008.070306

4 Simons FE. 9. Anaphylaxis. J Allergy Clin Immunol 2008; I2I(Suppl 2):S402-7. doi:10.1016/j.jaci.2007.08.061

5 Brown SG. Clinical features and severity grading of anaphylaxis. J Allergy Clin Immunol 2004; I 14:37I-6. doi:I0.1016/j.jaci.2004.04.029

6 Pumphrey RS. Lessons for management of anaphylaxis from a study of fatal reactions. Clin Exp Allergy 2000; 30:1।44-50. doi:I0.1046/j.I365-2222.2000.00864.x

7 Soar J, Pumphrey R, Cant A et al.; Working Group of the Resuscitation Council (UK). Emergency treatment of anaphylactic reactions - guidelines for healthcare providers. Resuscitation 2008 ; 77:157-69. doi:10.1016/j.resuscitation.2008.02.001

8 McLean-Tooke AP, Bethune CA, Fay AC et al. Adrenaline in the treatment of anaphylaxis: what is the evidence? BMJ 2003; 327:I332-5. doi:I0.II36/bmj.327.7427.1332

9 O'Driscoll BR, Howard LS, Davison AG on behalf of the British Thoracic Society. Guideline for emergency oxygen use in adult patients. Thorax 2008; 63:vil-68. doi:I0.II36/thx.2008.102947

10 Fisher MM. Clinical observations on the pathophysiology and treatment of anaphylactic cardiovascular collapse. Anaesth Intensive Care 1986; |4:|7-2|.

II British Thoracic Society, Scottish Intercollegiate Guidelines Network. British guideline on the management of asthma. Thorax 2008; 63:ivI-12I. doi:I0.I|36/thx.2008.09774I

12 Sheikh A, ten Broek V, Brown SG et al. HI-antihistamines for the treatment of anaphylaxis with and without shock. Cochrane Database Syst Rev 2007; I:CD006I60.

13 Schwartz LB. Diagnostic value of tryptase in anaphylaxis and mastocytosis. Immunol Allergy Clin North Am 2006; 26:45I-63. doi:10.1016/j.iac.2006.05.010 


\section{SELF-ASSESSMENT QUESTIONS}

I. A 29-year-old asthmatic is stung by a bee and rapidly develops tongue swelling, wheeze and stridor. The decision to give adrenaline is made. Which one of the following is the correct dose and route of administration?

A. $0.5 \mathrm{ml}(0.5 \mathrm{mg})$ I: 1000 adrenaline intravenous (IV).

B. $0.5 \mathrm{ml}(0.5 \mathrm{mg})$ I: 1000 adrenaline intramuscular (IM).

C. $0.5 \mathrm{ml}(0.5 \mathrm{mg}) \mathrm{I}: 1000$ adrenaline IM, followed by a repeat dose five minutes later if no better.

D. I mg I:10,000 adrenaline IV.

E. I mg I: 10,000 adrenaline IV, followed by a repeat dose 5 minutes later if no better.

2. A 32-year-old medical registrar with no medical history notices that her lips and face are swelling up. She is worried she has a serious allergy. Her airway, breathing and circulation are normal. Which one of the following should the attending emergency doctor do?

A. Administer adrenaline immediately.

B. Observe her for a period of time without giving any medications and examine her closely (especially skin, oropharynx and respiratory system).

C. Observe and examine her as in (B) but give chlorpheniramine and prednisolone as well.

D. Say she can go back to her respiratory ward round.

E. Take a serum tryptase to see if this is early anaphylaxis.
3. Concerning anaphylaxis, which one of the following is true?

A. The incidence of anaphylaxis in males and females is equal.

B. Food substances account for half the causes of anaphylaxis in the UK.

C. A single tryptase level at six hours confirms the diagnosis.

D. Anaphylaxis is most common in childhood.

E. Urticaria is the most common sign of anaphylaxis.

4. A 60-year-old female non-smoker is admitted with pneumonia. She is treated with amoxicillin but quickly develops signs in keeping with anaphylaxis. After adrenaline, which one of the following should occur?

A. She should receive a dose of steroids to prevent an acute relapse.

B. She should receive oxygen to maintain saturations at 94-98\%.

C. She should receive skin prick testing in the future.

D. She should be discharged with an emergency adrenaline device.

E. She should be more closely observed for two hours.

This paper was originally published as part of the Acute Medicine module in the RCPE Online Continuing Medical Education

Programme. Online CME, including the anwers to these questions, is available to Fellows and Members at: $h t t p: / / w w w . r c p e . a c . u k$

\section{CONTINUING MEDICAL EDUCATION (CME)}

\section{Online distance learning and self-assessment from the RCPE}

To allow users to update their general medical knowledge, the online CME modules contain a variety of material, including articles, interactive cases and quizzes, webstreamed lectures and self-assessment multiple choice questions (MCQs). Each module also includes material of particular relevance to those undertaking core medical training.

CME modules are eligible for external distance-learning Continuing Professional Development (CPD) credits under the UK Federation of Royal Colleges of Physicians CPD scheme. To claim these credits you must take the MCQ test for the module. Full instructions are given with each module.

The College's online CME programme is available to Fellows, Collegiate Members, Associates and e-Associates. Log on to the Fellows' and Members' secure area at: http://www.rcpe.ac.uk. If you have lost or forgotten your username or password please contact: webmaster@rcpe.ac.uk

\section{Currently available for CPD points}

- Cardiology (February 2010)

- Psychiatry (September 2009)

- Acute Medicine (April 2009) 